\title{
Site-specific cancer risk in patients with type 2 diabetes: a nationwide population-based cohort study in Korea
}

Suk Kyeong Kim ${ }^{1,2}$, Ju-Young Jang ${ }^{2}$, Dong-Lim Kim,2, Young A Rhyu ${ }^{2}$, Suh Eun Lee ${ }^{3}$, Seung-Hyun $\mathrm{Ko}^{4}$, Kyungdo Han ${ }^{5}$, Kee-Ho Song, ${ }^{1,2}$, and on behalf of the Task Force Team for Diabetes Fact Sheet of the Korean Diabetes Association

\begin{abstract}
${ }^{1}$ Division of Endocrinology and Metabolism, Department of Internal Medicine, Konkuk University School of Medicine, Seoul; ${ }^{2}$ Division of Endocrinology and Metabolism, Department of Internal Medicine, Konkuk University Medical Center Seoul; ${ }^{3}$ Graduate School of Medicine, Konkuk University, Seoul; ${ }^{4}$ Division of Endocrinology and Metabolism, Department of Internal Medicine, College of Medicine, The Catholic University of Korea, Seoul; ${ }^{5}$ Department of Biostatistic, The Catholic University of Korea, Seoul, Korea
\end{abstract}

\author{
Received: December 5, 2017 \\ Revised : March 8, 2018 \\ Accepted: April 1, 2018

\section{Correspondence to} \\ Kee-Ho Song, M.D. \\ Division of Endocrinology and \\ Metabolism, Department of \\ Internal Medicine, Konkuk \\ University School of Medicine, \\ 120-1 Neungdong-ro, Gwangjin- \\ $\mathrm{gu}$, Seoul 05030, Korea \\ Tel: $+82-2-2030-7533$ \\ Fax: +82-2-2030-7748 \\ E-mail:skh2k@kuh.ac.kr
}

Background/Aims: We aimed to evaluate site-specific cancer risk in diabetic patients and to investigate causal and temporal relationships by analyzing organ-specific cancer risk according to the duration of diabetes.

Methods: Using a database provided by the Korean National Health Insurance Service, we conducted a retrospective, population-based cohort study of adults aged $\geq 30$ years from January 2005 to December 2013. To verify the possibility of detection bias or reverse causation, we compared hazard ratios (HRs) for each cancer according to the following duration of diabetes: less than 6 months, 6 months to 3 years, and more than 3 years.

Results: The incidence of overall cancer per 1,000 person-years was higher in patients with diabetes than in those without diabetes (20.36 vs. 10.83). The overall cancer risk according to the duration of diabetes was the highest within the first 6 months after diagnosis (HR, 2.03; 95\% confidence interval [CI], 1.99 to 2.07 ), and the HR decreased with the duration of diabetes, ranging from 1.19 (95\% CI, 1.18 to 1.21) between 6 months and 3 years to 1.12 (95\% CI, 1.11 to 1.13) after 3 years. Both overall cancer risk and HR remained significantly higher in patients with diabetes than in those without diabetes. The risk for prostate cancer was higher in men with diabetes than in those without diabetes (HR, 1.12; 95\% CI, 1.10 to 1.14). In women, the risk for endometrial cancer was significantly higher in patients with diabetes than in those without diabetes throughout the duration of diabetes.

Conclusions: The risk for stomach, colorectum, liver, pancreas, and kidney cancer appeared to be higher in patients with diabetes than in those without diabetes regardless of the sex or duration of diabetes.

Keywords: Diabetes; Neoplasms; Korean National Health Insurance Service system

\section{INTRODUCTION}

The worldwide prevalence of diabetes has dramatically increased owing to the westernization of diet and sedentary lifestyle, and the increased incidence of diabetes mellitus is more pronounced in developing countries, including Asian countries [1-4]. This phenomenon has also been observed in epidemiological studies in Koreans $[5,6]$. Furthermore, an increase in the impaired fasting glucose owing to obesity and aging of the popu- 
lations will accelerate this phenomenon in the foreseeable future. Many previous studies have demonstrated an increased incidence of and mortality from cancer in subjects with diabetes [7-11]. Increased endogenous insulin levels resulting from insulin resistance, hyperglycemia, chronic inflammation, and increased oxidative stress have been suggested to be the main mechanism responsible for increased cancer risk in patients with diabetes $[10,12]$. Given the increased risk for diabetes and cancer in obese patients, obesity is thought to play a role not only in the onset of diabetes but also in the development of cancer in patients with diabetes [1216]. Because cancer develops over a long period, it is difficult to judge whether there is a simple association or a direct causal relationship between increased cancer incidence and diabetes [10]. De Bruijn et al. [17] suggested that detection bias based on findings, such as the highest incidence of cancer in the first 3 months after diagnosis and the gradual decrease afterward, results in a negligible difference in the risk between patients with and without diabetes. Therefore, it is necessary to determine whether the association between diabetes and cancer is causally related or whether there is detection bias or reverse causation by studying the temporal relationship between diabetes and cancer risk through population-based cohort studies [18].

Many of the existing studies have been conducted on Western populations with different genetic and environmental backgrounds from that of Koreans, and the results cannot be applied directly to Koreans. For example, the risk for prostate cancer in patients with diabetes is reduced in Western populations, while in Asian populations, including Koreans, it is known to increase $[16,19,20]$. This discrepancy implies that genetic and/ or environmental factors should always be considered in the interpretation of the results of these studies [21]. The Korean National Health Insurance Service (KNHIS) system (i.e., program, structure, medical coverage, types of insurance benefits, reimbursement flow, and healthcare delivery system) and the population and contents of the KNHIS database have been reported previously [22]. Briefly, the Korean National Health Insurance system run by government agencies like the NHIS and the Health Insurance Review Agency covers the entire population as a social insurance benefits scheme [22]. The NHIS database contains basic data such as age, sex, living area, and income level, as well as information about diagnosis statements as determined by the International Classification of Diseases 1oth revision (ICD10), the detailed statement of prescriptions, in-hospital administration, and surgery. Because the KNHIS database provides data on prevalence or incidence of disease, comorbidity, behavioral patterns of health care utilization, medical cost, and mortality of the entire Korean population, it can be used for population-based, nationwide studies of various diseases.

In this study, we aimed to investigate site-specific cancer risk of Korean patients aged $\geq 30$ years with diabetes and to estimate causal and temporal relationships by analyzing organ-specific cancer risk according to the duration of diabetes.

\section{METHODS}

\section{Data source}

Using a database provided by the KNHIS, we enrolled subjects aged $\geq 30$ years without diabetes mellitus between January 2005 and December 2007 and followed up to the development of cancer or until December 2013. The diabetes group included patients diagnosed with type 2 diabetes mellitus for the first time between January 2005 and December 2007. Subjects diagnosed with diabetes or cancer before January 2005, those diagnosed with diabetes after cancer, and those with missing data were excluded from the analysis. The control group was defined as all patients who did not have the exclusion criteria and who were not diagnosed with diabetes between January 2005 and December 2007 (Fig. 1).

As previously reported, the epidemiology committee of the Korean Diabetes Association approved the suitability of the dataset by validating its accuracy [5,23]. This study was approved by Institutional Review Board of the Korean National Institute for Bioethics Policy (Po1-201504-21-005) and was exempted from informed consent.

\section{Definition of diabetes and cancers}

We defined a person with diabetes as a subject who had ICD-10 codes of E11, E12, E13, or E14 as either a principal diagnosis or first to fourth additional diagnosis and who was prescribed one or more antidiabetic medica- 
tions for a given years [24]. A cancer case was defined as the presence of an ICD-10 code of ' $\mathrm{C}$ ' and an admission history with the cancer code as the principal diagnosis.

\section{Statistical analyses}

All analyses were performed using SAS version 9.2 (SAS Institute, Cary, NC, USA). Data are expressed as mean \pm standard deviation, numbers, and proportions (\%). An incident cancer case was defined as the first time the cancer was diagnosed, and the point was defined as the end point of the patient. We calculated total and site-specific cancer incidences and person-time incidence rates and compared these parameters with respect to sex and age. The hazard ratio (HR) for each cancer was calculated using Cox regression and adjusted for age and sex (model 1) or age, sex, income, place, hypertension, hyperlipidemia, chronic liver disease, ischemic heart disease, and chronic kidney disease (model 2). To verify the possibility of detection bias or reverse causation, we compared the HRs of each cancer according to the following duration of diabetes: < 6 months, 6 months to 3 years, and $\geq 3$ years. We also compared adjusted HRs for each cancer after excluding all patients with a duration of diabetes of $<6$ months. A $p<0.05$ was considered statistically significant.

\section{RESULTS}

\section{Baseline characteristics}

A total of 25,709,497 patients were enrolled, and the mean follow-up period was 8.6 years. The mean age was higher in the diabetes group $(58.0 \pm 12.6$ years $)$ than in the non-diabetes group $(47.3 \pm 13.1$ years), and the proportion of patients with hypertension, dyslipidemia, ischemic heart disease, and chronic kidney disease was higher in the diabetes group than in the non-diabetes group (Table 1).

\section{Total and site-specific cancer incidence and HRs}

The incidence of total cancer per 1,000 person-years was higher in the diabetes group than in the non-diabetes group (20.36 vs. 10.83) (Fig. 1). A similar tendency was observed when the incidence was compared according to sex, age, and individual cancer (Table 2). Table 3 shows HRs for any or individual cancer risks in the diabetes group, which were adjusted for age and sex (model 1) or age, sex, income, place, hypertension, hyperlipidemia, chronic liver disease, ischemic heart disease, and chronic kidney disease (model 2). The overall risk for cancer was 1.22 times higher (model 2) in patients with diabetes than in those without diabetes and more prominent in men (1.24) than in women (1.18). Oropharyngeal and esophageal cancers were not different between men in the diabetes and non-diabetes groups but were significantly decreased in women with diabetes. The risk for laryngeal cancer was 1.21 times higher in the men with diabetes, but was not different between women with diabetes and without diabetes. HRs for stomach, colorectal, liver, pancreas, lung, kidney, bladder, and thyroid cancers as well as leukemia were significantly higher in the diabetes group than in the non-diabetes group even after adjustment for age and sex (model 1) or age, sex, income, place, hypertension, hyperlipidemia, chronic liver disease, ischemic heart disease, and chronic kidney disease (model 2). Risk for prostate cancer (HR, 1.24; 95\% confidence interval [CI], 1.22 to 1.27) was higher in men with diabetes than in those without diabetes. HRs for breast, cervical, and endometrial cancers were significantly higher in the women with diabetes than in those without diabetes at 1.07, 1.06, and 1.34, respectively. Ovarian cancer was not different between the two groups (Table 3).

\section{HRs for total and site-specific cancer stratified by duration of diabetes}

The overall cancer risk was the highest in patients with a duration of diabetes of $<6$ months (HR, 2.03; 95\% CI, 1.99 to 2.07), but thereafter the magnitude of the HR tended to decrease over time, ranging from 1.19 (95\% CI, 1.18 to 1.21 ) between 6 months and 3 years to 1.12 (95\% CI, 1.11 to 1.13) after 3 years. Overall cancer risk and HRs remained significantly higher in the diabetes group than in the non-diabetes group over the entire study period. The HR of cancer was significantly higher in the pancreas, followed by the liver, leukemia, bladder, kidney, colorectum, larynx, thyroid, lung, and stomach in the diabetes group than in the non-diabetes group after being adjusted (HR from 1.98 to 1.13) (Table 3). The risks for cancer of the stomach, colorectum, liver, and pancreas remained higher in both sexes in the diabetes group than in the non-diabetes group for the entire du- 
Table 1. Baseline characteristics of study population

\begin{tabular}{|c|c|c|c|}
\hline \multirow{2}{*}{ Characteristic } & \multirow{2}{*}{ Total } & \multicolumn{2}{|c|}{ Diabetes mellitus } \\
\hline & & No & Yes \\
\hline Number & $25,709,497$ & $25,011,562$ & 697,935 \\
\hline Age, $\mathrm{yr}^{\mathrm{a}}$ & $47 \cdot 7 \pm 13.2$ & $47 \cdot 3 \pm 13.1$ & $58.0 \pm 12.6$ \\
\hline \multicolumn{4}{|l|}{ Age distribution, $\mathrm{yr}^{\mathrm{a}}$} \\
\hline $30-39$ & $8,435,222(32.81)$ & $8,384,679(33 \cdot 52)$ & $50,543(7.24)$ \\
\hline $40-49$ & $7,720,250(30.03)$ & $7,575,744(30.29)$ & $144,506(20.7)$ \\
\hline $50-59$ & $4,571,710(17.78)$ & $4,386,280(17.54)$ & $185,430(26.57)$ \\
\hline $60-69$ & $2,931,055(11.40)$ & $2,754,796(11.01)$ & $176,259(25.25)$ \\
\hline$\geq 70$ & $2,051,260(7.98)$ & $1,910,063(7.64)$ & $141,197(20.23)$ \\
\hline Male sex & $12,540,261(48.78)$ & $12,160,000(48.61)$ & $383,133(54.9)$ \\
\hline Place, urban & $12,231,992(47.58)$ & $11,882,897(47 \cdot 51)$ & $349,095(50.02)$ \\
\hline \multicolumn{4}{|l|}{ Income } \\
\hline Beneficiaries & $689,101(2.68)$ & $610,202(2.44)$ & $78,899(11.3)$ \\
\hline Q1 & $6,298,481(24 \cdot 50)$ & $6,131,082(24 \cdot 51)$ & $167,399(23.98)$ \\
\hline Q2 & $6,160,855(23.96)$ & $6,017,568(24.06)$ & $143,287(20.53)$ \\
\hline Q3 & $6,170,051(24.00)$ & $6,027,405(24.1)$ & $142,646(20.44)$ \\
\hline $\mathrm{Q}_{4}$ & $6,391,009(24.86)$ & $6,225,305(24.89)$ & $165,704(23.74)$ \\
\hline Hypertension $^{\mathrm{a}}$ & $3,091,949(12.03)$ & $2,829,819(11.31)$ & $262,130(37.56)$ \\
\hline Dyslipidemia $^{\mathrm{a}}$ & $880,529(3.42)$ & $778,356(3.11)$ & $102,173(14.64)$ \\
\hline Cardiovascular disease $^{\mathrm{a}}$ & $994,494(3.87)$ & $934,953(3.74)$ & $59,541(8.53)$ \\
\hline Chronic kidney disease ${ }^{\mathrm{a}}$ & $63,355(0.25)$ & $56,476(0.23)$ & $6,879(0.99)$ \\
\hline Ischemic heart disease $^{\mathrm{a}}$ & $848,992(3.30)$ & $782,467(3.13)$ & $66,525(9.53)$ \\
\hline Follow-up duration, yr & $8.6 \pm 1.5$ & $8.6 \pm 1.4$ & $7.1 \pm 1.7$ \\
\hline
\end{tabular}

Values are presented as mean \pm SD or number (\%).

${ }^{a} p<0.005$ between diabetics and non-diabetics.

ration of diabetes. Only men with diabetes showed an increased risk for leukemia and cancers of the larynx, lung, kidney, bladder, and thyroid during the entire duration of diabetes. The risk for oropharyngeal and esophageal cancer was higher in patients with a duration of diabetes of $<6$ months but significantly lower in patients with diabetes with a duration of $\geq 3$ years. The risk for prostate cancer was higher in men with diabetes than in those without diabetes (HR, 1.12; 95\% CI, 1.10 to 1.14). The cancer risk was not significantly different between women with diabetes and those without diabetes over the duration of diabetes between 6 months and 3 years (larynx, lung, and kidney cancer) or $\geq 3$ years (larynx, bladder, thyroid cancer, and leukemia). The risk for breast, cervical, and ovarian cancer was higher in patients with diabetes with a duration of $<6$ months but not in those with a longer duration of diabetes. The risk for endometrial cancer was significantly higher in the diabetes group than in the non-diabetes group for the entire study duration of diabetes (Table 4).

\section{HRs for the risk for total and specific cancer except diabetics with a duration of $<6$ months}

Except for patients with diabetes with a duration of $<6$ months who showed the highest risk for cancer incidence, we recalculated the HRs for overall and individual cancers adjusted for age and sex (model 1) or age, sex, income, place, hypertension, hyperlipidemia, chronic liver disease, ischemic heart disease, and chronic kidney disease (model 2) (Table 5). The overall risk for cancer incidence increased in both men and women with diabetes regardless of model 1 or model 


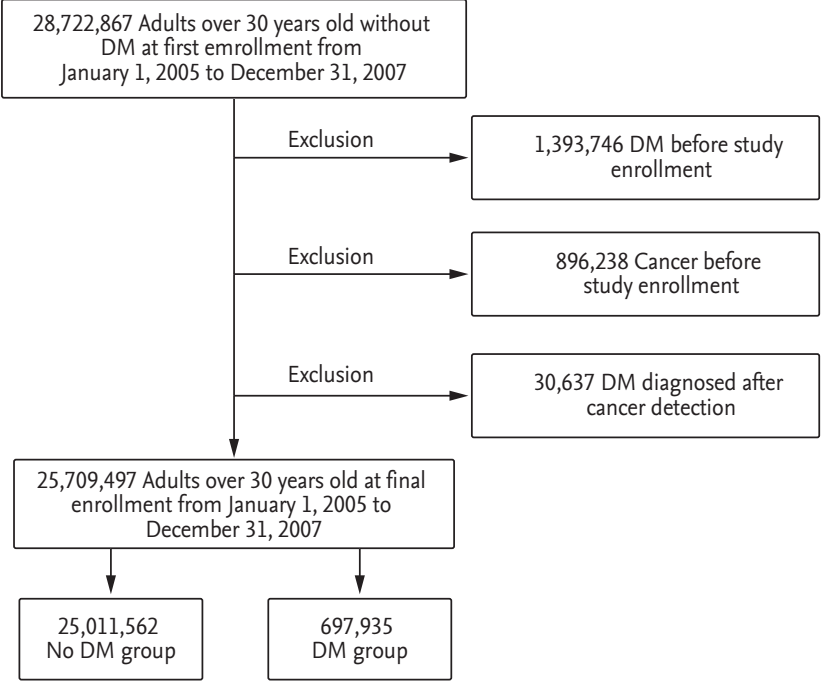

Figure 1. Study population. We excluded subjects who were diagnosed with diabetes or cancer before January 2005, those diagnosed with diabetes after cancer, and those with missing data from analysis. DM, diabetes mellitus.

2. The risk for stomach, colorectal, liver, pancreatic, kidney, bladder, and thyroid cancer as well as leukemia remained increased in the diabetes group. Oropharyngeal and esophageal cancer were not different in men with or without diabetes, but the risk was significantly decreased in women with diabetes.

Larynx and lung cancer showed an increased risk in men with diabetes only. The risk for prostate cancer in men with diabetes and the risk for endometrial cancer in women with diabetes were statistically significantly higher than in men and women without diabetes.

\section{DISCUSSION}

We found that the incidence of cancer was higher in patients with diabetes than in those without diabetes, and this phenomenon was noticeable within 6 months of the diagnosis of diabetes. The risks for cancer of the stomach, colorectum, liver, and pancreas remained higher in both sexes in the diabetes group than in the non-diabetes group for the entire duration of diabetes.

The risk for cancer in patients with diabetes varies according to the duration of the disease, location of cancer, and sex of the patient. In particular, the risk for pancreatic cancer was notably higher in patients with diabetes shortly after diagnosis (duration $<6$ months; 5.94-fold) than in those without diabetes; however, it rapidly decreased over time. Nevertheless, the fact that a 1.64-fold increase in risk persisted for $\geq 3$ years suggests that diabetes is an important risk factor for pancreatic cancer, despite detection bias in the early period. A similar tendency was observed in cancers involving the colorectum, prostate, and endometrium. During the entire duration of diabetes, our results were consistent with those of previous studies [11,12,18,25-27], showing increased incidences of pancreatic, liver, colorectal, prostate, and endometrial cancer in patients with diabetes. It is possible that reverse causation and detection bias may have played a role in the seemingly increased incidence of cancer immediately after the diagnosis of diabetes; however, because the risk remained high after 6 months duration of diabetes, we can interpret the result as a causal relationship.

Additionally, the increase in cancer incidence of the stomach, colorectum, liver, pancreas, prostate, and endometrium was statistically significant even after the adjustment for age, sex, underlying disease, and socioeconomic status, which supports our claim that there is a causal relationship between diabetes and cancer in certain organs. Although the risk for larynx, lung, kidney, thyroid, and bladder cancer was significantly increased in both sexes even after adjusting for sex, underlying disease, and socioeconomic status, an analysis based on the duration of the disease yielded different results: a significant increase in risk was observed in all duration groups for men, whereas a varying significance was observed in women depending on the duration, implying a difference between the sexes. The difference in the risk for larynx and lung cancer in Korea between men and women may be related to the low smoking rate of women.

Numerous debates are ongoing regarding whether the relationship between diabetes and prostate cancer is positive or negative or whether the relationship exists at all [28]. One of the prominent features of the relationship between diabetes and prostate cancer is that it appears to differ among ethnicities. In prospective studies, retrospective studies, and meta-analyses that targeted mainly Caucasians, the prostate cancer incidence was lower in patients with diabetes than in those without diabetes, whereas studies involving the Asian 


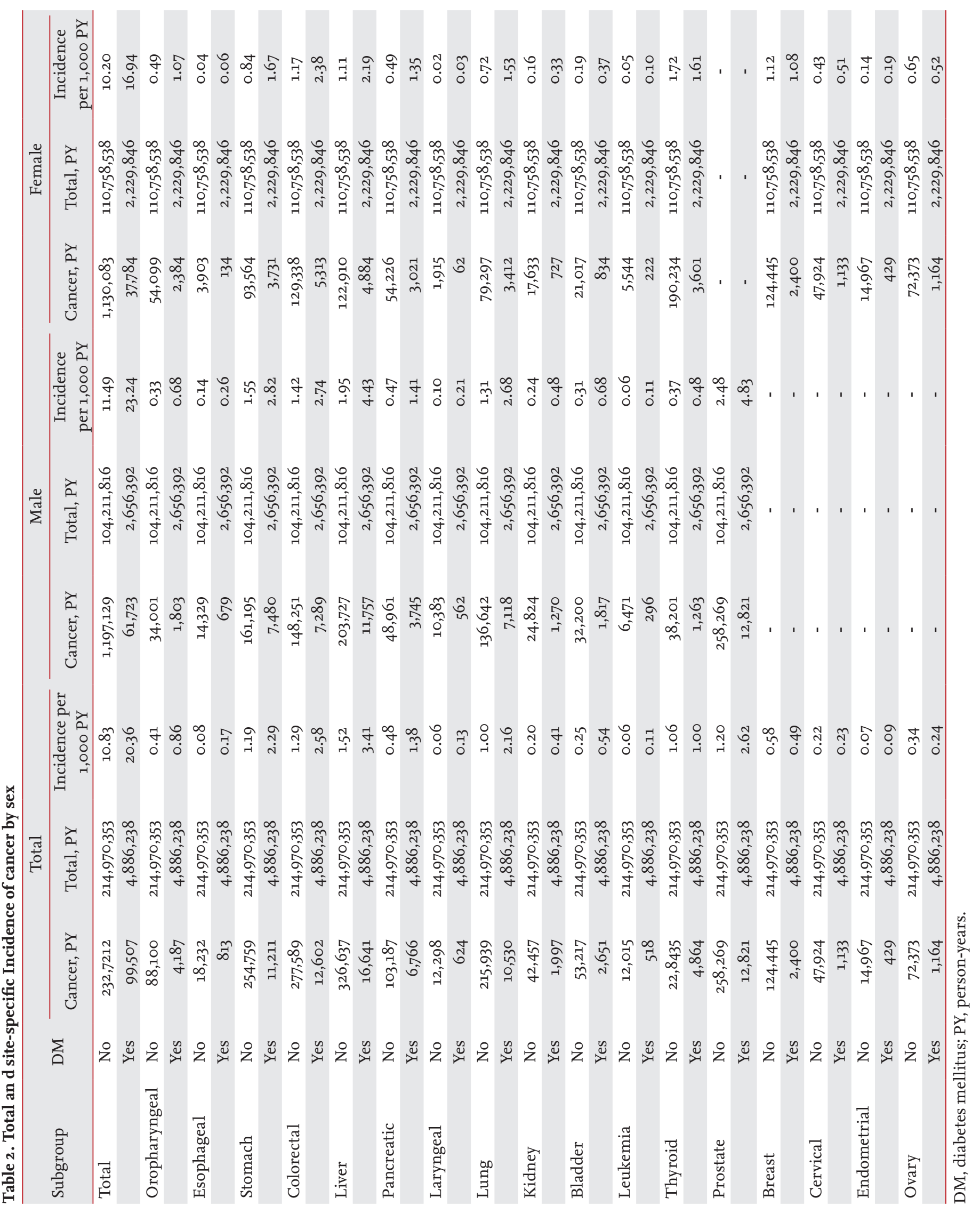


Table 3. HRs for the risk of total and specific cancer in diabetes group

\begin{tabular}{|c|c|c|c|c|c|c|}
\hline & \multicolumn{6}{|c|}{$\mathrm{HR}(95 \% \mathrm{CI})$} \\
\hline & \multicolumn{2}{|c|}{ Total } & \multicolumn{2}{|c|}{ Male } & \multicolumn{2}{|c|}{ Female } \\
\hline & Model $1^{a}$ & Model $2^{b}$ & Model $1^{a}$ & Model $2^{b}$ & Model $1^{a}$ & Model $2^{b}$ \\
\hline Total & $1.31(1.30-1.32)^{\mathrm{c}}$ & $1.22(1.21-1.22)^{\mathrm{C}}$ & $1.36(1.34-1.37)^{c}$ & $1.24(1.23-1.25)^{\mathrm{c}}$ & $1.26(1.25-1.28)^{\mathrm{c}}$ & $1.18(1.17-1.20)$ \\
\hline Oropharyngeal & $1.08(1.04-1.11)^{c}$ & $0.97(0.94-1.00)$ & $1.16(1.11-1.22)^{\mathrm{c}}$ & $1.05(1.00-1.10)$ & $1.02(0.98-1.07)$ & $0.92(0.88-0.96)^{d}$ \\
\hline Esophageal & $1.02(0.95-1.10)$ & $0.97(0.90-1.04)$ & $1.08(1.00-1.16)$ & $1.02(0.95-1.11)$ & $0.83(0.70-1.00)$ & $0.76(0.64-0.91)^{d}$ \\
\hline Stomach & $1.16(1.14 \cdot 1.18)^{c}$ & $1.13(1.10-1.15)^{c}$ & $1.15(1.13-1.18)^{c}$ & $1.12(1.10-1.15)^{c}$ & $1.18(1.14-1.22)^{c}$ & $1.14(1.10-1.18)^{c}$ \\
\hline Colorectal & $1.28(1.26-1.31)^{c}$ & $1.21(1.18-1.23)^{\mathrm{c}}$ & $1.29(1.26-1.33)^{\mathrm{c}}$ & $1.21(1.19-1.24)^{\mathrm{c}}$ & $1.28(1.24-1.32)^{\mathrm{c}}$ & $1.20(1.16-1.23)^{\mathrm{c}}$ \\
\hline Liver & $1.73(1.71-1.76)^{c}$ & $1.43(1.41-1.45)^{\mathrm{c}}$ & $1.84(1.80-1.87)^{c}$ & $1.49(1.46-1.51)^{\mathrm{c}}$ & $1.52(1.48-1.57)^{c}$ & $1.33(1.29-1.37)^{\mathrm{c}}$ \\
\hline Pancreatic & $2.12(2.07-2.18)^{c}$ & $1.98(1.93-2.03)^{c}$ & $2.22(2.15-2.30)^{c}$ & $2.06(1.99-2.13)^{\mathrm{c}}$ & $2.02(1.94-2.09)^{c}$ & $1.88(1.81-1.96)^{c}$ \\
\hline Laryngeal & $1.25(1.15-1.35)^{\mathrm{c}}$ & $1.18(1.08-1.28)^{c}$ & $1.28(1.18-1.40)^{\mathrm{c}}$ & $1.21(1.11-1.32)^{\mathrm{c}}$ & $1.03(0.80-1.33)$ & $0.95(0.73-1.22)$ \\
\hline Lung & $1.19(1.16-1.21)^{c}$ & $1.14(1.12-1.16)^{\mathrm{c}}$ & $1.21(1.18-1.24)^{\mathrm{c}}$ & $1.17(1.15-1.20)^{\mathrm{c}}$ & $1.15(1.11-1.19)^{c}$ & $1.08(1.04-1.11)^{\mathrm{c}}$ \\
\hline Kidney & $1.45(1.38-1.52)^{c}$ & $1.24(1.18-1.30)^{\mathrm{c}}$ & $1.47(1.39-1.55)^{\mathrm{c}}$ & $1.26(1.19-1.34)^{\mathrm{c}}$ & $1.42(1.32-1.53)^{c}$ & $1.20(1.11-1.29)^{\mathrm{c}}$ \\
\hline Bladder & $1.37(1.32-1.43)^{\mathrm{C}}$ & $1.28(1.23-1.33)^{\mathrm{c}}$ & $1.40(1.33-1.47)^{\mathrm{c}}$ & $1.30(1.24-1.37)^{\mathrm{c}}$ & $1.35(1.25-1.44)^{c}$ & $1.23(1.14-1.32)^{c}$ \\
\hline Leukemia & $1.38(1.26-1.50)^{c}$ & $1.29(1.18-1.42)^{\mathrm{c}}$ & $1.33(1.18-1.49)^{\mathrm{c}}$ & $1.25(1.11-1.41)^{c}$ & $1.46(1.27-1.67)^{\mathrm{c}}$ & $1.36(1.18-1.56)^{c}$ \\
\hline Thyroid & $1.25(1.22-1.29)^{\mathrm{c}}$ & $1.17(1.14-1.21)^{\mathrm{c}}$ & $1.47(1.39-1.55)^{\mathrm{c}}$ & $1.36(1.28-1.44)^{c}$ & $1.19(1.15-1.23)^{\mathrm{c}}$ & $1.12(1.08-1.16)^{c}$ \\
\hline Prostate & $1.24(1.22-1.27)^{\mathrm{C}}$ & $1.12(1.10-1.14)^{\mathrm{c}}$ & $1.24(1.22-1.27)^{\mathrm{c}}$ & $1.12(1.10-1.14)^{\mathrm{c}}$ & - & - \\
\hline Breast & $1.10(1.05-1.14)^{c}$ & $1.07(1.03-1.11)^{\mathrm{c}}$ & - & - & $1.09(1.05-1.14)^{\mathrm{c}}$ & $1.07(1.03-1.11)^{\mathrm{c}}$ \\
\hline Cervical & $1.10(1.03-1.16)^{c}$ & $1.06(1.00-1.12)$ & - & - & $1.09(1.03-1.16)^{\mathrm{c}}$ & $1.06(1.00-1.12)$ \\
\hline Endometrial & $1.41(1.27-1.55)^{\mathrm{c}}$ & $1.34(1.21-1.48)^{\mathrm{c}}$ & - & - & $1.41(1.27-1.55)^{\mathrm{c}}$ & $1.34(1.21-1.48)^{\mathrm{c}}$ \\
\hline Ovary & $1.05(0.99-1.12)$ & $1.03(0.97-1.09)$ & - & - & $1.05(0.99-1.12)$ & $1.03(0.97-1.09)$ \\
\hline
\end{tabular}

HR, hazard ratio; CI, confidence interval.

${ }^{a}$ Model 1 were adjusted for age and sex.

${ }^{b}$ Model 2 were adjusted for age, sex, income, place, hypertension, hyperlipidemia, chronic liver disease, ischemic heart disease, and chronic kidney disease.

${ }^{\mathrm{c}}$ Significantly increased compared to non-diabetes group $(p<0.05)$.

${ }^{\mathrm{d}}$ Significantly decreased compared to non-diabetes group $(p<0.05)$.

populations showed a significant increase in prostate cancer risk in patients with diabetes $[19,20]$. Our findings are consistent with previous studies performed in the Asian populations, with a significantly increased risk for prostate cancer in patients with diabetes regardless of the duration of the disease. Statistical significance was retained even after adjustments were made for age, underlying disease, and socioeconomic status. This result supports the discrepancy in the interaction between risk factors for prostate cancer in patients with diabetes and carcinogenic environmental factors associated with diabetes among the populations owing to genetic background.

For oropharyngeal and esophageal cancer, the incidence risk increased for the first 6 months after diagnosing diabetes; however, after 3 years, the risk was lower in the diabetes group than in the non-diabetes group. This phenomenon was more prominent in women than in men. Additional long-term studies would be required to address the question of whether the duration of diabetes is negatively related to the incidence risk for these two cancers. However, detection bias may cause cancer incidence to appear to be increased in patients with diabetes. Breast and ovarian cancer showed similar results. The incidence risk significantly increased for the first 6 months after diagnosis, after which the difference became negligible, further supporting the existence of detection bias.

The strength of this study is that it is a large-scale epidemiological study involving the entire Korean pop- 


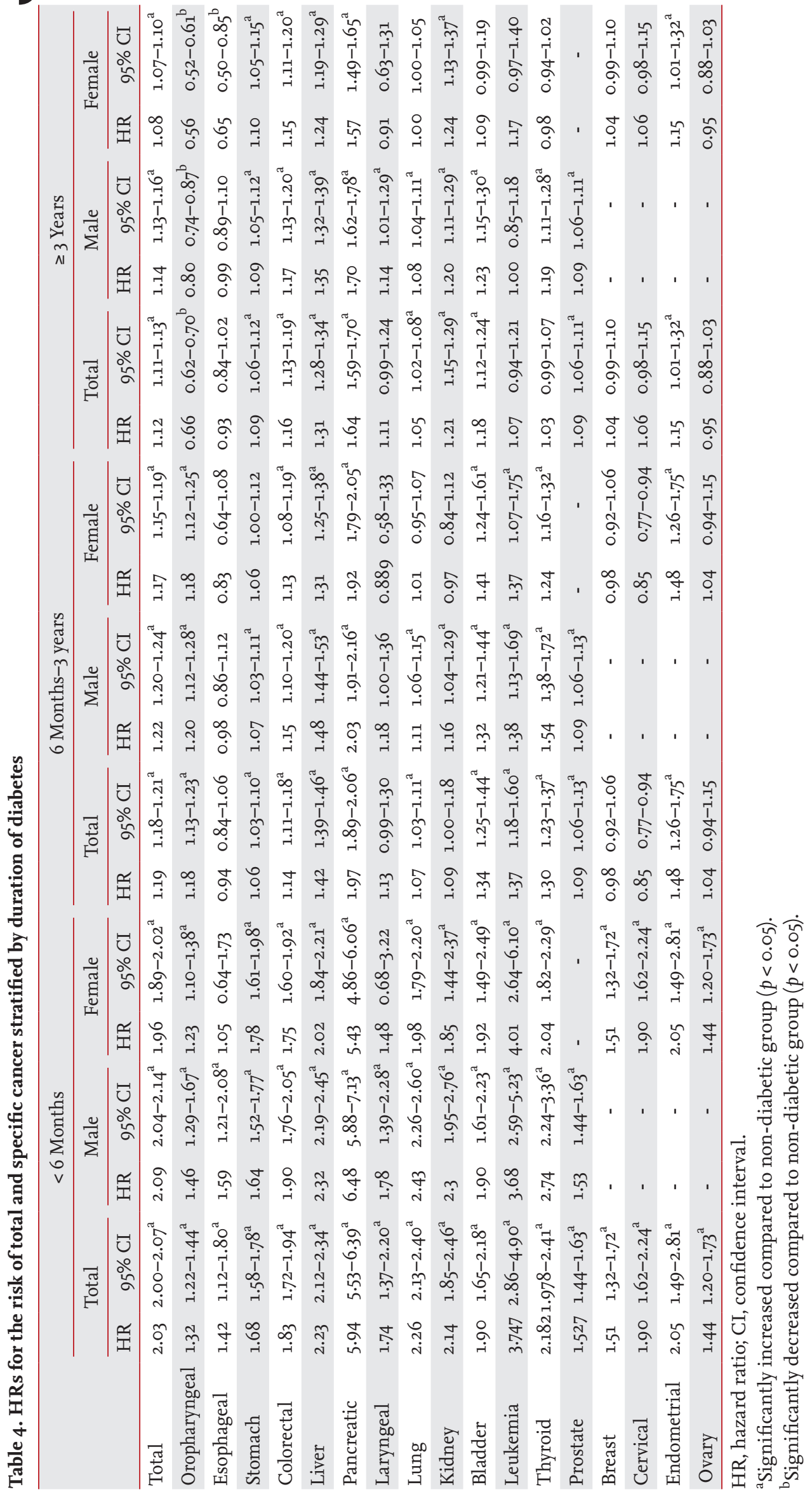


Table 5. HRs for the risk of total and specific cancer except diabetics with a duration of $<6$ months

\begin{tabular}{|c|c|c|c|c|c|c|}
\hline & \multicolumn{6}{|c|}{$\operatorname{HR}(95 \% \mathrm{CI})$} \\
\hline & \multicolumn{2}{|c|}{ Total } & \multicolumn{2}{|c|}{ Male } & \multicolumn{2}{|c|}{ Female } \\
\hline & Model $1^{a}$ & Model $2^{b}$ & Model $1^{a}$ & Model $2^{b}$ & Model $1^{a}$ & Model $2^{b}$ \\
\hline Total & $1.24(1.23-1.25)^{c}$ & $1.16(1.15-1.16)^{c}$ & $1.28(1.27-1.29)^{\mathrm{c}}$ & $1.18(1.17-1.19)^{\mathrm{c}}$ & $1.20(1.18-1.21)^{c}$ & $1.13(1.11-1.14)^{c}$ \\
\hline Oropharyngeal & $1.03(1.00-1.07)$ & $0.93(0.90-1.00)$ & $1.10\left(1.05^{-1.16}\right)^{c}$ & $1.00\left(0.95^{-1.05}\right)$ & $0.99(0.94-1.03)$ & $0.88(0.85-0.93)^{d}$ \\
\hline Esophageal & $0.99(0.92-1.06)$ & $0.94(0.87-1.01)$ & $1.04(0.96-1.12)$ & $0.99(0.91-1.08)$ & $0.80(0.66-0.96)^{d}$ & $0.73(0.61-0.89)^{d}$ \\
\hline Stomach & $1.11(1.09 \cdot 1.13)^{\mathrm{c}}$ & $1.08(1.06-1.11)^{c}$ & $1.11(1.08-1.13)^{c}$ & $1.08(1.06-1.11)^{\mathrm{c}}$ & $1.13(1.09-1.17)^{c}$ & $1.09(1.05-1.13)^{c}$ \\
\hline Colorectal & $1.23(1.21-1.26)^{c}$ & $1.16(1.14-1.18)^{c}$ & $1.24(1.21-1.27)^{c}$ & $1.17(1.14-1.20)^{\mathrm{c}}$ & $1.23(1.20-1.27)^{\mathrm{c}}$ & $1.16(1.12-1.19)^{c}$ \\
\hline Liver & $1.62(1.60-1.65)^{c}$ & $1.37(1.34-1.39)^{c}$ & $1.71(1.68-1.75)^{c}$ & $1.41(1.39-1.44)^{c}$ & $1.45(1.41-1.49)^{c}$ & $1.28(1.24-1.32)^{c}$ \\
\hline Pancreatic & $1.89(1.84-1.94)^{c}$ & $1.76(1.72-1.81)^{c}$ & $1.96(1.89-2.03)^{c}$ & $1.82(1.75-1.89)^{c}$ & $1.81(1.74-1.89)^{c}$ & $1.69(1.63-1.77)^{c}$ \\
\hline Laryngeal & $1.19(1.09-1.30)^{\mathrm{c}}$ & $1.13(1.03-1.23)^{c}$ & $1.23(1.12-1.34)^{c}$ & $1.16(1.06-1.27)^{c}$ & $0.99(0.76-1.30)$ & $0.91(0.69-1.19)$ \\
\hline Lung & $1.10(1.08-1.13)^{c}$ & $1.06(1.04-1.08)^{c}$ & $1.12(1.09-1.15)^{\mathrm{c}}$ & $1.09(1.06-1.12)^{c}$ & $1.08(1.04-1.12)^{\mathrm{c}}$ & $1.01(0.97-1.05)$ \\
\hline Kidney & $1.37(1.30-1.44)^{c}$ & $1.17(1.12-1.23)^{\mathrm{c}}$ & $1.38(1.29-1.46)^{\mathrm{c}}$ & $1.19(1.12-1.26)^{\mathrm{C}}$ & $1.36(1.26-1.47)^{c}$ & $1.15(1.06-1.25)^{c}$ \\
\hline Bladder & $1.33(1.27-1.38)^{\mathrm{c}}$ & $1.23(1.18-1.29)^{c}$ & $1.35(1.29-1.42)^{\mathrm{c}}$ & $1.26(1.20-1.33)^{c}$ & $1.30(1.21-1.40)^{c}$ & $1.19(1.10-1.28)^{\mathrm{c}}$ \\
\hline Leukemia & $1.24(1.13-1.36)^{c}$ & $1.18(1.07-1.29)^{c}$ & $1.19(1.05-1.35)^{c}$ & $1.14(1.00-1.29)$ & $1.32(1.14-1.52)^{c}$ & $1.24(1.07-1.43)^{c}$ \\
\hline Thyroid & $1.19\left(1.15^{-1.22}\right)^{\mathrm{c}}$ & $1.12(1.08-1.15)^{c}$ & $1.39(1.31-1.48)^{c}$ & $1.29(1.22-1.37)^{c}$ & $1.13(1.09-1.17)^{c}$ & $1.07(1.03-1.11)^{c}$ \\
\hline Prostate & $1.21(1.19-1.23)^{\mathrm{c}}$ & $1.09(1.07-1.12)^{\mathrm{c}}$ & $1.21(1.19-1.23)^{\mathrm{c}}$ & $1.09(1.07-1.12)^{c}$ & - & - \\
\hline Breast & $1.05(1.00-1.09)$ & $1.03(0.99-1.08)$ & - & - & $1.05(1.00-1.09)$ & $1.03(0.99-1.08)$ \\
\hline Cervical & $1.01\left(0.95^{-1.08)}\right.$ & $0.98(0.92-1.05)$ & - & - & $1.01\left(0.95^{-1.08)}\right.$ & $0.98(0.92-1.05)$ \\
\hline Endometrial & $1.35(1.22-1.49)^{\mathrm{c}}$ & $1.29(1.16-1.43)^{c}$ & - & - & $1.35(1.22-1.49)^{c}$ & $1.29(1.16-1.43)^{c}$ \\
\hline Ovary & $1.01(0.95-1.08)$ & $0.99(0.93-1.06)$ & - & - & $1.01(0.95-1.08)$ & $0.99(0.93-1.06)$ \\
\hline
\end{tabular}

HR, hazard ratio; CI, confidence interval.

${ }^{a}$ Model 1 were adjusted for age and sex.

${ }^{\mathrm{b}}$ Model 2 were adjusted for age, sex, income, place, hypertension, hyperlipidemia, chronic liver disease, ischemic heart disease, and chronic kidney disease.

${ }^{a}$ Significantly increased compared to non-diabetes group $(p<0.05)$.

${ }^{\mathrm{b}}$ Significantly decreased compared to non-diabetes group $(p<0.05)$.

ulation aged $\geq 30$ years. Thus far, this is the first study regarding diabetes and cancer incidence risk that encompasses $>25$ million subjects. This strength enables our study to provide comprehensive information on cancer risk in Korean patients with diabetes and to be used as important evidence in establishing guidelines for public health policies. Our study had some foreseeable drawbacks. First, because diabetes is defined in this study using certain disease codes plus the use of anti-diabetic medications, patients with initial-stage diabetes who are on a controlled diet and exercise plan but not using medications may have been excluded. However, a previous study [29] showed that this proportion of patients is very low (1.1\%), and because the results indicated a high incidence of cancer in the first
6 months after the diagnosis of diabetes, the limitation does not appear to have a profound effect on the findings of this study. Second, patients who were diagnosed with diabetes after January 1, 2008 were included in the non-diabetes group for analysis. However, because cancer incidence in patients with diabetes was the highest shortly after diagnosis and the time interval between January 2008 and December 2013 was relatively small, these factors may have caused the early stage increases in incidence to be more prominent, without further influencing the interpretation. Finally, as noted in a previous study [30], problems regarding the accuracy of diagnosis and the failure to address adjustments for risk factors related to each specific cancer owing to limited clinical and laboratory information remain. For 
example, cancer cases attributable to both diabetes and high body mass index were almost twice as frequent in women (especially breast and endometrial cancer) than in men (especially liver and colorectal cancer) in the independent scenario [31]. Because patients with diabetes tend to be obese, a limited portion of the increased cancer incidence in these patients can be attributed to obesity. However, we believe this factor needs to be allowed as a limitation of epidemiological studies using health insurance claim data [24,32].

In conclusion, the risk for cancer increases in patients with diabetes, and the phenomenon is more prominent over short periods, i.e., in the first 6 months after diagnosis. As the duration of diabetes increases, incidence risk for cancer varies depending on the site of cancer and the patient's sex. Thus, in patients with diabetes, cancer screening should be individualized based on the duration of diabetes, sex, and the location of cancer.

\section{KEY MESSAGE}

1. The incidence of total cancer per 1,000 person-years was higher in the diabetes group than in the control group.

2. The risks for cancer of the stomach, colorectum, liver, and pancreas remained higher in both sexes in the diabetes group than in the control for the entire duration of diabetes.

3. The risk for oropharyngeal and esophageal cancer was significantly higher in patients with diabetes with a duration of $<6$ months than in those with diabetes with a duration of $\geq 3$ years.

4. In contrast to reports that the risk for prostate cancer is decreased in Caucasians with diabetes, the risk for prostate cancer in Korean male patients with diabetes was higher than in those without diabetes.

5. The risk for endometrial cancer was significantly higher in women with diabetes than in those without diabetes for the duration of the disease.

\section{Conflict of interest}

No potential conflict of interest relevant to this article was reported.

\section{REFERENCES}

1. International Diabetes Federation. IDF Diabetes Atlas. 7th ed. Brussels (BE): International Diabetes Federation, 2015.

2. Li J, Dong Y, Wu T, Tong N. Differences between Western and Asian type 2 diabetes patients in the incidence of vascular complications and mortality: a systematic review of randomized controlled trials on lowering blood glucose. J Diabetes 2016;8:824-833.

3. Andersen GS, Kamper-Jorgensen Z, Carstensen B, Norredam M, Bygbjerg IC, Jorgensen ME. Diabetes among migrants in Denmark: incidence, mortality, and prevalence based on a longitudinal register study of the entire Danish population. Diabetes Res Clin Pract 2016;122:9-16.

4. Papier K, Jordan S, D'Este C, et al. Incidence and risk factors for type 2 diabetes mellitus in transitional Thailand: results from the Thai cohort study. BMJ Open 2016;6:0014102.

5. Ha KH, Kim DJ. Trends in the diabetes epidemic in Korea. Endocrinol Metab (Seoul) 2015;30:142-146.

6. Korean Diabetes Association. Korean Diabetes Fact Sheet 2015 [Internet]. Seoul (KR): Korean Diabetes Association, c2015 [cited 2017 Jul 30]. Available from: http://www. diabetes.or.kr/pro/news/admin.php?category=B\&code $=$ admin\&mode=view\&number $=1427$.

7. Magliano DJ, Davis WA, Shaw JE, Bruce DG, Davis TM. Incidence and predictors of all-cause and site-specific cancer in type 2 diabetes: the Fremantle Diabetes Study. Eur J Endocrinol 2012;167:589-599.

8. Oberaigner W, Ebenbichler C, Oberaigner K, Juchum M, Schonherr HR, Lechleitner M. Increased cancer incidence risk in type 2 diabetes mellitus: results from a cohort study in Tyrol/Austria. BMC Public Health 2014;14:1058.

9. Giovannucci E, Harlan DM, Archer MC, et al. Diabetes and cancer: a consensus report. Diabetes Care 2010;33:1674-1685.

10. Vigneri P, Frasca F, Sciacca L, Pandini G, Vigneri R. Diabetes and cancer. Endocr Relat Cancer 2009;16:1103-1123.

11. Renehan AG, Yeh HC, Johnson JA, et al. Diabetes and cancer (2): evaluating the impact of diabetes on mortality in patients with cancer. Diabetologia 2012;55:1619-1632.

12. Szablewski L. Diabetes mellitus: influences on cancer risk. Diabetes Metab Res Rev 2014;30:543-553. 
13. Lauby-Secretan B, Scoccianti C, Loomis D, et al. Body fatness and cancer: viewpoint of the IARC working group. N Engl J Med 2016;375:794-798.

14. Font-Burgada J, Sun B, Karin M. Obesity and cancer: the oil that feeds the flame. Cell Metab 2016;23:48-62.

15. Guevara-Aguirre J, Rosenbloom AL. Obesity, diabetes and cancer: insight into the relationship from a cohort with growth hormone receptor deficiency. Diabetologia 2015:58:37-42.

16. Garg SK, Maurer H, Reed K, Selagamsetty R. Diabetes and cancer: two diseases with obesity as a common risk factor. Diabetes Obes Metab 2014;16:97-110.

17. De Bruijn KM, Ruiter R, de Keyser CE, Hofman A, Stricker BH, van Eijck CH. Detection bias may be the main cause of increased cancer incidence among diabetics: results from the Rotterdam Study. Eur J Cancer 2014;50:2449-2455.

18. Johnson JA, Carstensen B, Witte D, et al. Diabetes and cancer (1): evaluating the temporal relationship between type 2 diabetes and cancer incidence. Diabetologia 2012;55:1607-1618.

19. Bansal D, Bhansali A, Kapil G, Undela K, Tiwari P. Type 2 diabetes and risk of prostate cancer: a meta-analysis of observational studies. Prostate Cancer Prostatic Dis 2013;16:151-158.

20. Pierce BL. Why are diabetics at reduced risk for prostate cancer? A review of the epidemiologic evidence. Urol Oncol 2012;30:735-743.

21. Liu JH, Li HW, Tong M, Li M, Na YQ. Genetic risk factors of prostate cancer in Han nationality population in Northern China and a preliminary study of the reason of racial difference in prevalence of prostate cancer. Zhonghua Yi Xue Za Zhi 2004;84:364-368.

22. Song SO, Jung CH, Song YD, et al. Background and data configuration process of a nationwide population-based study using the Korean National Health Insurance system. Diabetes Metab J 2014;38:395-403.

23. Song SO, Lee YH, Kim DW, et al. Trends in diabetes incidence in the last decade based on Korean national health insurance claims data. Endocrinol Metab (Seoul) 2016;31:292-299.

24. Lee YH, Han K, Ko SH, Ko KS, Lee KU; Taskforce Team of Diabetes Fact Sheet of the Korean Diabetes Association. Data analytic process of a nationwide population-based study using national health information database established by National Health Insurance Service. Diabetes Metab J 2016;40:79-82.

25. Suh S, Kim KW. Diabetes and cancer: is diabetes causally related to cancer? Diabetes Metab J 2011;35:193-198.

26. Lega IC, Wilton AS, Austin PC, Fischer HD, Johnson JA, Lipscombe LL. The temporal relationship between diabetes and cancer: a population-based study. Cancer 2016;122:2731-2738.

27. Nath SD, Habib SL, Abboud HE. Fasting serum glucose level and cancer risk in Korean men and women. JAMA 2005;293:2210-2211.

28. Crawley D, Chamberlain F, Garmo H, et al. A systematic review of the literature exploring the interplay between prostate cancer and type two diabetes mellitus. Ecancermedicalscience 2018;12:802.

29. Jeon JY, Kim DJ, Ko SH, et al. Current status of glycemic control of patients with diabetes in Korea: the fifth Korea National Health and Nutrition Examination Survey. Diabetes Metab J 2014;38:197-203.

30. Ko SH, Kim DJ, Park JH, et al. Trends of antidiabetic drug use in adult type 2 diabetes in Korea in 2002-2013: nationwide population-based cohort study. Medicine (Baltimore) 2016;95:e4018.

31. Pearson-Stuttard J, Zhou B, Kontis V, Bentham J, Gunter MJ, Ezzati M. Worldwide burden of cancer attributable to diabetes and high body-mass index: a comparative risk assessment. Lancet Diabetes Endocrinol 2018;6:95-104.

32. Koo BK, Moon MK. Are we in the same risk of diabetes mellitus? Gender- and age-specific epidemiology of diabetes in 2001 to 2014 in the Korean population. Diabetes Metab J 2016;40:175-181. 